\title{
La importancia de la evaluación del neurodesarrollo en niños menores de treinta meses en el contexto peruano
}

\section{The importance of the neural development assessment in children aged less than $\mathbf{3 0}$ months in the Peruvian context}

Correspondencia

Ericson Gutierrez

eringunza@yahoo.es

Recibido: 01/06/2016

Aprobado: 20/07/2016

Citar como: Gutierrez EL, Lazarte F, Alarcon G. La importancia de la evaluación del neurodesarrollo en niños menores de treinta meses en el contexto pervano. Acta Med Peru. 2016;33(4):304-8

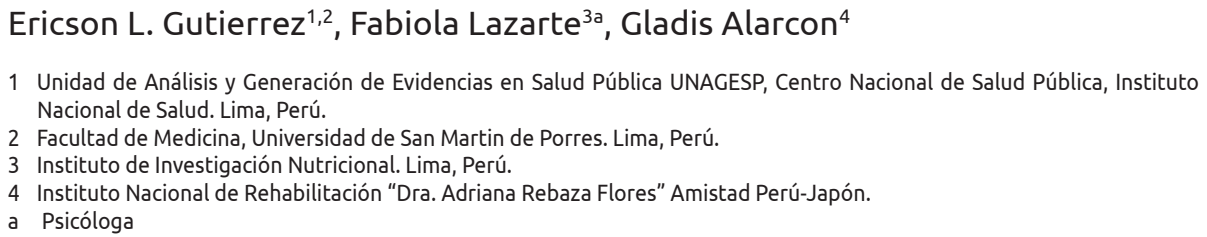

\section{RESUMEN}

La evaluación del neurodesarrollo es una actividad básica en los servicios de atención del niño. Las pruebas que evalúan el neurodesarrollo infantil se dividen en dos grupos, las de detección o tamizaje y las de diagnóstico o evaluación. Las de detección fueron diseñadas para identificar a los niños en riesgo, utilizándose en una primera etapa para recomendar una futura evaluación. Posteriormente se utilizan las pruebas de diagnóstico que son aplicadas con mayor detalle por especialistas en el área. Una vez identificado el niño en riesgo de retraso es necesario que sea incluido oportunamente en un programa de intervención, principalmente antes de los 3 años. La prevención de un posible retraso y la recuperación de los niños en riesgo contribuirán a la reducción de la pobreza y la inequidad, ya que los niños podrán adquirir capacidades y destrezas que le aseguren una adecuada calidad de vida para ellos y sus familias contribuyendo con la economía y el desarrollo de país.

Palabras clave: Desarrollo infantil; Desempeño psicomotor; Pruebas neuropsicológicas (fuente: DeCS BIREME).

\section{ABSTRACT}

Assessing the neural development is a basic activity in child health services. The tests that assess the neural development in children are divided in two groups: screening tests and diagnostic or assessment tests. Screening tests were designed for identifying children at risk, and they are used in a first-step fashion in order to recommend further testing. Afterwards, diagnostic tests are used, which are administered by specialists in this field. Once the child at risk for retardation in his/her neural development is identified, it is necessary that he/she may be enrolled in an intervention program, especially before three years of age. Prevention of such cases and timely therapy and recovery of children at risk will contribute to reduce poverty and inequity, since these children may acquire abilities and skills that will ensure adequate quality of life for them and their families, also contributing with the economy and development of this country.

Keywords: Child Development; Psychomotor performance; Neuropsychological tests (source: MeSH NLM). 


\section{INTRODUCCIÓN}

El neurodesarrollo es un proceso de adquisición de conocimientos, habilidades, actitudes y funciones que se adquieren continuamente a lo largo de la vida ${ }^{[1]}$. La proliferación de las dendritas y la mielinización de los axones son, desde el punto de vista fisiológico, los responsables de este proceso; por lo tanto, el neurodesarrollo representa la manifestación externa de la maduración del sistema nervioso central ${ }^{[2]}$.

La mayor parte de este proceso se da entre la etapa fetal y los primeros tres años de vida. En este periodo las conexiones de los circuitos cerebrales se forman rápidamente y de forma muy eficaz. A pesar de que la actividad cerebral está influenciada por patrones genéticos, el cerebro en esta etapa, tiene la suficiente plasticidad para cambiar su organización y sus funciones en base a las experiencias y estímulos ambientales. Es así como llamamos a estos primeros años, la ventana de oportunidad. Las situaciones adversas en la infancia temprana pueden producir daños fisiológicos en estos circuitos, los cuales persisten hasta la etapa adulta y probablemente sean irreversibles ${ }^{[3]}$.

Existen factores que favorecen un adecuado neurodesarrollo, como una adecuada suplementación materna en el embarazo, una buena alimentación del niño ${ }^{[4]}$, un sólido vínculo madre-hijo y una estimulación sensorial adecuada y oportuna ${ }^{[5]}$.

Los factores que interfieren con el neurodesarrollo son aquellas condiciones que pueden producir un daño neuronal irreversible como son: una hipoxia mantenida, la hipoglicemia, y las infecciones o traumatismos del SNC ${ }^{[5]}$.

En el Perú aún existen muchas personas viviendo en situación de pobreza y extrema pobreza, lo cual conlleva a tener un mayor riesgo de vivir en condiciones adversas que afectan su desarrollo integral, entre ellas tenemos la malnutrición, falta de cuidado prenatal, infecciones, complicaciones al nacer, maltrato, entre otros.

Existe evidencia de que los niños con retardo en el crecimiento tienen un mayor riesgo de presentar déficits en su desarrollo cognitivo y más adelante educativo, el cual se manifiesta en mayor deserción escolar y menor rendimiento escolar ${ }^{[6]}$. Se estima que los déficits acumulados, en el desarrollo cognitivo y educacional, por parte de los niños con desnutrición crónica resultan en una disminución de un $22 \%$ de pérdidas en sus ingresos anuales en la adultez ${ }^{[7]}$.

La presente revisión tiene como objetivo describir la importancia de la detección temprana de alguna alteración del neurodesarrollo en los niños menores de 30 meses, así como describir los principales test utilizados para su evaluación por personal de salud.

\section{Importancia de la detección temprana de posibles retrasos del neurodesarrollo}

Aproximadamente el $80 \%$ del desarrollo cerebral ocurre hasta los tres años de edad, y entre los tres y seis años se establecen las capacidades básicas, especialmente el habla y la capacidad de comunicación ${ }^{[8]}$.

Por lo tanto, en la actualidad se busca evaluar y detectar problemas en el neurodesarrollo antes de cumplir esa edad ya que se considera más beneficioso que el niño participe en programas de intervención lo antes posible, en función al nuevo conocimiento que se tiene sobre la plasticidad cerebral y los periodos críticos o sensibles a la intervención ${ }^{[9]}$. De esta manera, detectar y dar un tratamiento oportuno a niños que presentan algún grado de deficiencia en su desarrollo repercutirá en el resto su vida, en su bienestar futuro y calidad de vida.

\section{Escalas utilizadas en la evaluación del neurodesarrollo en níños menores de 30 meses en el Perú}

Existen diferentes tipos de prueba que evalúan el desarrollo infantil. Se pueden dividir en dos grandes grupos, las de diagnóstico, que tienen como objetivo evaluar de manera detallada el desarrollo de un niño y las de detección o tamizaje. Las de diagnóstico tienen propiedades psicométricas de validez, fiabilidad, sensibilidad y especificidad, lo cual permite identificar a niños en situación de riesgo o retraso en el desarrollo infantil [10]. La evaluación del desarrollo con este tipo de pruebas es realizada por psicólogos especializados con capacitación en el uso de instrumentos de evaluación de este tipo.

Las Escalas Bailey de Desarrollo Infantil o el Inventario de Desarrollo de Battelle en su forma completa constituyen ejemplos de pruebas diagnósticas que han sido administradas a grandes muestras de evaluación en países como Chile, Colombia, México y Perú ${ }^{[10]}$.

El segundo tipo de pruebas, son las llamadas, según Schady y Berlinski ${ }^{[10]}$, pruebas de detección. Están diseñadas para identificar a niños en situación de riesgo. Se caracterizan más por su sensibilidad que por su especificidad. Se utilizan en una primera etapa de diagnóstico, algunas de ellas son llamadas pruebas de tamizaje. Su administración es más fácil, contienen menos ítems y en su mayoría la información se obtiene del reporte materno más que el de la observación directa de los niños. Un ejemplo de estas pruebas El ASQ (Cuestionario de Edades y Etapas), la prueba de Detección del Inventario de Desarrollo de Battelle, la Prueba de Tamizaje del Desarrollo de Denver, la escala de Nelson Ortiz, la Prueba Nacional de Pesquisa (PRUNAPE) y el test peruano del desarrollo del niño y la niña menor de 30 meses. Estas pruebas han sido administradas a grandes muestras de evaluación en Argentina, Brasil, Colombia, Ecuador, Honduras, México, Nicaragua y Perú.

Dentro de las limitaciones de los test de evaluación, apreciamos que es difícil conocer su validez pronostica debido a que existen numerosas variables que intervienen en el desarrollo infantil ${ }^{[11]}$.

La decisión de utilizar un tipo de prueba o la otra se tiene que hacer en base al objetivo de la evaluación, la edad del niño del niño, los recursos disponibles y el perfil de las personas que lo vayan a aplicar $^{[11]}$. 
En el 2010, el Ministerio de Salud del Perú aprobó la Norma Técnica de Salud (NTS) Nº 087 - MINSA/DGSP - V.01 "Norma Técnica de Salud para el Control y Desarrollo de la Niña y el Niño menor de 5 años" mediante la Resolución Ministerial $N^{\circ}$ 990-2010/MINSA; en este documento se establecieron las disposiciones para la evaluación oportuna y periódica del crecimiento y desarrollo del niño menor de cinco años ${ }^{[2]}$. En la mencionada norma técnica se establece como instrumento de medición del neurodesarrollo del niño el Test Peruano del Desarrollo del Niño y la Niña Menor de 30 meses. Este test es considerado uno de detección o tamizaje, como se mencionó anteriormente.

\section{Instrumentos de evaluación del neurodesarrollo comúnmente utilizados en el país}

Se expondrán tres test de tamizaje, el Test Peruano del Desarrollo del Niño y la Niña Menor de 30 meses, utilizado por norma en los establecimientos de salud del Perú, la Escala de Evaluación del Desarrollo Psicomotor, el cual también es utilizado comúnmente por personal de salud del sector público y el Ages and Stages Questionnaire, utilizado como instrumento de medición para el programa Nacional Cuna Más. En cuanto a los test de diagnóstico solo se expondrá sobre el test de Bayley, debido a que es considerado el gold standard internacional, así mismo porque ha sido utilizado en estudios de investigación en el Perú.

\section{Test Peruano del desarrollo del niño y la niña menor de 30 meses}

Este test fue elaborado por el Instituto Nacional de Rehabilitación (INR) y viene siendo aplicado en niños peruanos de forma regular. El esquema de test que el INR planteó, colocó hitos que tuvieran en buena parte de ellos una correlación neurológica que le dé una mayor sensibilidad a la prueba para la detección de lesiones orgánicas en forma precoz.

Este test consta de doce ítems correspondientes a los diferentes comportamientos, se encuentran en el eje de las ordenadas o columna de la izquierda del instrumento, estos son: comportamiento motor postural (tres ítems), comportamiento viso motor (dos ítems), comportamiento del lenguaje (tres ítems), comportamiento personal-social (tres ítems) e inteligencia-aprendizaje (un ítem). En el eje de las abscisas; o fila superior, está colocada la edad cronológica del niño, mes por mes durante el primer año y luego a los 15, 18, 21, 24 y 30 meses de edad.

En la experiencia diaria utilizando esta prueba, se aprecia que el test aplicado por un profesional debidamente entrenado puede tener una duración de 20 minutos. En cuanto a la aceptación de los padres de familia y de los niños evaluados, según la experiencia, el test es amigable y motivador para el niño, permitiendo mantener su atención durante la evaluación ${ }^{[33]}$.

\section{La Escala de Evaluación del Desarrollo Psicomotor (EEDP)}

La EEDP es una prueba de tamizaje que fue creada en Chile en 1974 con el fin de evaluar el desarrollo global de los/as niños/as, no solo en el área motora, sino en las diferentes dimensiones del desarrollo infantil como los aspectos de lenguaje, cognitivos, sociales y físicos. Se aplica en infantes de entre o y 24 meses de edad y está diseñado para captar alteraciones o variaciones en el desarrollo psicomotor.

Esta escala fue estandarizada mediante una muestra de 600 niños, estratificando la muestra entre el sexo, el nivel socioeconómico y la presumible normalidad del niño. Para establecer la confiabilidad del instrumento se aplicó el testretest, encontrando que el EEDP tenía una alta consistencia entre sus ítems.

Este instrumento consta de 52 ítems en cuatro áreas del desarrollo: lenguaje, motora, social y coordinación, y tiene por objetivo establecer un coeficiente de edad mental y de desarrollo [14]. Los resultados obtenidos con este test se categorizan en tres: normal, en riesgo y retraso.

La EEDP es comúnmente usada en el Perú, pues su utilización fue establecida en establecimientos de salud antes de la incorporación del test peruano del desarrollo del niño y la niña en la norma técnica del MINSA ${ }^{[15]}$.

\section{Ages and Stages Questionnaire (ASO)}

Es un cuestionario que evalúa el desarrollo psicomotor de los niños a través del reporte de los padres o cuidadores principales, de la observación directa de los niños o una combinación de ambos. Fue desarrollado en los años 80 y revisado en el 2009, culminando en su tercera versión ( $\mathrm{ASO}_{3}$ ). Está diseñado para dos usos: i) para ser aplicado por los padres en el hogary realizar una verificación inicial de que el desarrollo de sus hijos se está dando sobre los parámetros esperados, antes de acudir a un especialista, y ii) en el contexto de un programa de desarrollo infantil, para monitorear a niños que están en riesgo de sufrir discapacidades o retrasos en su desarrollo. La literatura sugiere que este instrumento es una herramienta apropiada para el tamizaje en diversos contextos, así mismo ha sido utilizado en países latinoamericanos como Brasil, Ecuador y Perú ${ }^{[16]}$. El ASO consta de una serie de veinte cuestionarios que corresponden a periodos de desarrollo según la edad desde el nacimiento hasta los 6 años.

Este instrumento ha sido utilizado para establecer la línea de base y evaluaciones posteriores en cuanto al desarrollo psicomotor de los niños beneficiarios del programa Nacional Cuna Más en el año $2012{ }^{[17]}$. Cada cuestionario contiene 30 preguntas que están clasificadas en cinco áreas o dimensiones de desarrollo (seis preguntas por dimensión).

\section{Bayley Scale of Infant Development (BSID)}

El gold standard establecido internacionalmente en el caso de los instrumentos de medición del desarrollo infantil es el BSID. Este test fue desarrollado por Nancy Bayley en 1969 en un principio para niños entre el nacimiento y los 30 meses (BSID I). Posteriormente se revisa en el año 1984 (BSID II) y por último en 1993 se extendió su rango de aplicación hasta 
los 42 meses (BSID III) ${ }^{[18]}$. Las versiones anteriores a las del Bayley III arrojan un índice de desarrollo mental y un índice de desarrollo motor. También evalúan el desarrollo social y emocional a través del reporte materno. A diferencia de sus predecesoras, el BSID III incluye subescalas (cognitiva, lenguaje, motory socio-emocional) que pueden ser puntuadas por separado de manera que si se quiere evaluar un dominio especifico puede hacerse.

Este test ha sido traducido en una gran cantidad de países desarrollados y en vías de desarrollo, y la mayoría de los instrumentos que se han creado posteriormente, se han comparado con él, al analizar sus propiedades psicométricas.

Fernald at al. ${ }^{[19]}$ declara que es muy sensible a diferentes tipos de intervenciones y que los puntajes del Bayley han sido correlacionados fuertemente con el test de habilidades académicas en niños de mayor edad como las escalas Mc Carthy de habilidades del niño, las ascalas Weschler, entre otras. Además, estudios longitudinales en la etapa infantil donde los niños han sido evaluados con el test de Bayley han sido fuertemente asociados a un nivel de educación en la adultez.

EI BSID II ha sido utilizado con éxito en población peruana por investigadores del Instituto de Investigación nutricional ${ }^{[20]}$. La diversidad del material del kit del test puede hacer a esta prueba adaptable a cualquier contexto.

\section{Identificación del niño con retraso}

Un estudio demostró que utilizando solo la evaluación clínica del médico se habría dejado de detectar hasta un $45 \%$ de niños elegibles para intervención. Por esta razón es necesaria la aplicación periódica y constante de instrumentos de evaluación estandarizados ${ }^{[21]}$.

En los Estados Unidos solo el 23\% de los médicos de atención primaria utilizan estos test de evaluación. La limitación más importante reportada fue la falta de tiempo, incluyendo también el aumento de costos, necesidad de personal entrenado y la falta de consenso sobre los test a utilizar ${ }^{[21]}$.

Recientemente en nuestro país se están realizando evaluaciones para determinar el porcentaje de profesionales de la salud que aplican de forma correcta el test peruano del desarrollo en establecimientos de salud del primer nivel de atención ${ }^{[22]}$. Estos resultados serán importantes para conocer la línea basal y plantear intervenciones para mejorar posibles deficiencias.

La actual norma técnica vigente (NTS $N^{\circ} 087$ - MINSA/DGSP V.01) establece una serie de actividades que realizará el personal de salud, de acuerdo a los resultados obtenidos en la evaluación del neurodesarrollo. Estas van desde explicar las pautas para una adecuada estimulación hasta la referencia del niño a un establecimiento de mayor complejidad.

\section{CONCLUSIONES}

De acuerdo a lo expuesto previamente, la valoración del neurodesarrollo es una actividad básica en la atención de la etapa de vida niño. La detección temprana de cualquier retraso, utilizando adecuadamente los test de evaluación contribuye a un tratamiento precoz, minimizando posibles secuelas.

La evaluación del neurodesarrollo cumple con todas las características para realizar un sistema de screening poblacional debido a su importancia en Salud Pública, y a que el riesgo de retraso es relativamente prevalente y que existen tratamientos de intervención temprana disponibles ${ }^{[23]}$.

Las actividades que comprenden la evaluación del niño sano, la estimulación temprana, la detección y tratamiento del retraso requieren de inversión estatal; tanto en la captación, retención y capacitación de profesionales de la salud, así como en la implementación de programas de estimulación temprana e intervención oportuna en casos de algún rezago del desarrollo.

La evidencia científica sugiere que la inversión estatal en el desarrollo infantil es la más costo-efectiva que se puede realizar. Algunas estimaciones establecen que por cada dólar invertido hay un retorno de hasta US\$17 dólares ${ }^{[24]}$. Esto se debe a que como adulto productivo genera ingresos más altos y representa menores costos en servicios sociales.

Así, cuanto mayor es la proporción de personas con capacidades para ser productivas, mayor es la competitividad que tiene la sociedad en la economía global. Por ello, la sociedad debe establecer las condiciones necesarias para que todas las personas que pertenecen a ella puedan adquirir esas capacidades que aseguren un buen nivel de calidad de vida para ellas y sus dependientes.

\section{Fuente de financiamiento: Instituto Nacional de Salud}

Declaración de conflicto de intereses: Los autores declaran no tener conflicto de intereses con la publicación de este artículo.

\section{REFERENCIAS BIBLIOGRÁFICAS}

1. Carey WB, Crocker AC, Elias ER, Feldman HM, Coleman WL. Developmental-Behavioral pediatrics: expert consult. 4 th ed. Philadelphia: Saunders Elsevier Health Sciences; 2009.

2. Rodriguez M. Patología prenatal con manifestaciones neurológicas en el período neonatal. Neurol Inf Argentina. 2011;2(3):13-20.

3. Victora C. Los mil días de oportunidad para intervenciones nutricionales. De la concepción a los dos años de vida. Arch Argent Pediatr. 2012;110(4):311-7.

4. Anjos T, Altmäe S, Emmett $P$, Tiemeier $H$, Closa-Monasterolo $R$, Luque $V$, et al. Nutrition and neurodevelopment in children: focus on NUTRIMENTHE project. Eur J Nutr. 2013;52(8):1825-42.

5. Moore R. Evaluación del desarrollo psicomotor. En: Guiraldes Cameratti E, Ventura-Junca P (ed). Manual de Pediatría [Internet]. Santiago, Chile: Pontificia Universidad Católica de Chile; 2002 [citado el 30 de diciembre de 2016]. p.1-3. Disponible en: http:// 
escuela.med.puc.cl/paginas/publicaciones/manualped/evaldessps. $\mathrm{html}$

6. PollittT, Caycho T. Desarrollo motor como indicador del desarrollo infantil durante los primeros dos años de vida. Revista de Psicología. 2010;28(2):385-413.

7. Grantham-McGregor S, Cheung YB, Cueto S, Glewwe P, Richter $L$, Strupp B, et al. Developmental potential in the first 5 years for children in developing countries. Lancet. 2007;369(9555):60-70.

8. Equipo Gerencial del PRIDI. Programa Regional de Indicadores de Desarrollo Infantil (PRIDI). Marco conceptual [Internet]. New York; Banco Interamericano de Desarrollo; 2011 [citado el 30 de diciembre de 2016]. Disponible en: http://www.iadb.org/ education/pridi/instrumentos/Marco_Conceptual.pdf

9. Fernandez-De-Cossio L, Brugues S, Álvarez M. Pesquisaje de problemas del neurodesarrollo, pruebas disponibles y las funciones ejecutivas. Cuadernos Sociales. 2011;11(1):99-119.

10. Schady S, Berlinsky N (ed). Los primeros años. El bienestar infantil y el papel de las políticas públicas [Internet]. New York; Banco Interamericano de Desarrollo; 2015 [citado el 30 de diciembre de 2016]. Disponible en: https://publications.iadb.org/bitstream/ handle/11319/7259/Los_primeros_a\%C3\%B10s_El_bienestar_ infantil_y_el_papel_de_las_pol\%C_\%ADticas_p\%C3\%BAblicas. pdf? sequence $=1$

11. Ruiz G. Diagnóstico funcional en primera infancia: Utilidad y límites de las escalas/tests psicométricos. Desenvolupa. 2012;(33):1-14.

12. Ministerio de Salud, Dirección General de Salud de las Personas. Norma técnica de salud para el control y desarrollo de la niña y el niño menor de cinco años. Lima, Perú: Ministerio de Salud; 2011.

13. Instituto Nacional de Rehabilitación. Test Peruano del desarrollo del niño y la niña [Internet]. Lima: Instituto Nacional de Rehabilitación [citado el 30 de diciembre de 2016]. Disponible en: http://www3. inr.gob.pe/Docencia/fotos\%2ode\%2olibros/489_MINSA14-1.pdf

14. Ministerio de Salud. Escala de Evaluación del Desarrollo Psicomotor de o- 24 meses [Internet]. Lima, Perú: Ministerio de Salud; 1995 [citado el 30 de diciembre de 2016]. Disponible en: http://bvs. minsa.gob.pe/local/minsa/2333.PDF
15. Martinez C, Urdangarin D. Evaluación del desarrollo psicomotor de niños institucionalizados menores de 1 año mediante tres herramientas distintas de evaluación. [Tesis para optar el Título de Kinesiología]. Santiago, Chile: Universidad de Chile; 2005.

16. Kyerematen $\mathrm{V}$, Hamb A, Oberhelman RA, Cabrera L, BernabeOrtiz A, Berry SJ. Exploratory application of the Ages and Stages (ASO) child development screening test in a low-income Peruvian shantytown population. BMJ Open. 2014;4(1):e004132.

17. Ministerio de Inclusión Social. Nota metodológica para la evaluación de impacto del programa CUNA MÁS. Modalidad acompañamiento a familias [Internet]. Lima, Perú: Ministerio de Inclusión Social; 2011 [citado el 30 de diciembre de 2016]. Disponible en: http://www.midis.gob.pe/dgsye/evaluacion/ documentos/CUNAMAS-AF_30_de_Enero.pdf

18. Weiss LG, Oakland T, Aylward GP. Bayley-III Clinical Use and Interpretation. San Diego, United States: Academic Press; 2010.

19. Fernald LCH, Kariger $P$, Engle $P$, Raikes $A$. Examining early child development in low-income countries: a toolkit for the assessment of children in the first five years of life. Washington D.C.: The World Bank; 2009.

20. Colombo J, Zavaleta N, Kannass KN, Lazarte F, Albornoz C, Kapa LL, et al. Zinc Supplementation Sustained Normative Neurodevelopment in a Randomized, Controlled Trial of Peruvian Infants Aged 6-18 Months. J Nutr. 2014;144(8):1298-305.

21. Mackrides PS, Ryherd SJ. Screening for developmental delay. Am Fam Physician. 2011;84(5):544-9.

22. Instituto Nacional de Estadística e Informática (INEI). Encuesta a establecimientos de salud en la atención del control de crecimiento, desarrollo y vacunas [Internet]. Lima: INEI; c2016 [citado el 30 de diciembre de 2016]. Disponible en: http://www. inei.gob.pe/estadisticas/encuestas/

23. Iceta A, Yoldi L. Desarrollo psicomotor del niño y su valoración en atención primaria. ANALES Sis San Navarra. 2002;25 Supl 2:35-43.

24. Heckman J, Dimitriy VM. The productivity argument for investing in young children. Working Paper No. 5. Arlington, VA: Committee for Economic Development; 2004.

\section{Ahora puede enviar sus artículos para Acta Médica Peruana en nuestro Open Journal System:}

\section{www.amp.cmp.org.pe}

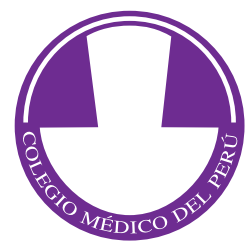

